\title{
Precision lattice calculation of SU(2) 't Hooft loops
}

\author{
Philippe de Forcrand \\ Institut für Theoretische Physik, ETH Hönggerberg, CH-8093 Zürich, Switzerland \\ and CERN, Physics Department, TH Unit, CH-1211 Geneva 23, Switzerland
}

David Noth

Paul Scherrer Institut, CH-5232 Villigen PSI, Switzerland

(Received 16 June 2005; published 2 December 2005)

\begin{abstract}
The [dual] string tension of a spatial 't Hooft loop in the deconfined phase of Yang-Mills theory can be formulated as the tension of an interface separating different $Z_{N}$ deconfined vacua. We review the 1-loop perturbative calculation of this interface tension in the continuum and extend it the lattice. The lattice corrections are large. Taking these corrections into account, we compare Monte Carlo measurements of the dual string tension with perturbation theory, for $\mathrm{SU}(2)$. Agreement is observed at the $2 \%$ level, down to temperatures $\mathcal{O}(10) T_{c}$.
\end{abstract}

DOI: 10.1103/PhysRevD.72.114501

PACS numbers: 11.15.Ha, 12.38.Gc, 11.10.Wx, 12.38.Mh

\section{INTRODUCTION}

Because of asymptotic freedom, the running coupling $g(T)$ in an $\mathrm{SU}(N)$ Yang-Mills theory becomes small at high temperature. However, a perturbative calculation of $\mathrm{SU}(N)$ thermodynamic properties faces two obstacles. $g(T)$ only runs logarithmically, so that subleading orders of the perturbative expansion contribute significantly as the temperature is lowered towards $T_{c}$, the confinement/deconfinement transition temperature. And infrared divergences prevent an analytic, perturbative treatment altogether beyond some order. Heroic efforts have been devoted to the calculation of the pressure to this maximum order $g^{6}$ [1]. The expansion converges poorly. One may fear that this is a general feature, and that an accurate perturbative calculation of any thermodynamic property is only possible at astronomically high temperatures. The purpose of this paper is to show a counterexample to this pessimistic view: the spatial 't Hooft loop.

The tension of a spatial 't Hooft loop has been calculated in perturbation theory to the first three nontrivial orders, and the expansion appears to converge fast [2,3]. We show here that this perturbative calculation agrees with nonperturbative Monte Carlo measurements in the SU(2) theory to a high precision, down to temperatures $\mathcal{O}(10) T_{c}$.

The 't Hooft loop has not received the same attention as the Wilson loop in numerical simulations of $\mathrm{SU}(N)$ lattice gauge theories. There are several causes for this relative neglect.

(i) First, as 't Hooft has shown [4], area law or perimeter law for Wilson and 't Hooft loops together is forbid- den, in the absence of massless modes. As a result, in $\mathrm{SU}(N)$ Yang-Mills theory, an area law is only observed for the spatial 't Hooft loop (dual to the temporal Wilson loop) above the deconfinement temperature $T_{c}$. The associated dual string tension $\tilde{\sigma}$ serves as an order parameter for deconfinement $[5,6]$. The deconfined phase has traditionally received less attention than the confined phase, although the situation has been changing with the experimental search for the quark-gluon plasma.

(ii) Second, the intuitive, "physical" meaning of a spatial 't Hooft loop has not been widely appreciated, and it is often considered as an "exotic" observable of marginal interest. The fact is that a 't Hooft loop enforces a $Z_{N}$ interface in the Euclidean system, and that the dual string tension $\tilde{\sigma}$ is nothing else but the interface tension (up to a conventional factor $T$ ). This is easiest to see on the lattice. Starting from an Euclidean lattice of size $L_{x} \times L_{y} \times L_{z} \times L_{t}$, with lattice spacing $a\left(L_{i}=N_{i} a\right)$ and periodic boundary conditions in all directions, let us construct a rectangular't Hooft loop $\tilde{W}(\partial \tilde{\Sigma})$ in the $(x, y)$ plane. The contour $\partial \tilde{\Sigma}$, which we take rectangular for simplicity, is the boundary of a surface $\tilde{\Sigma}$, both on the dual lattice. Let us take for $\tilde{\Sigma}$ the minimal, planar surface, with coordinates $\left(z_{0}, t_{0}\right)$. Each plaquette of $\tilde{\Sigma}$ is dual to a $(z, t)$ plaquette. These $(z, t)$ plaquettes with fixed coordinates $\left(z_{0}, t_{0}\right)$ form a "stack" $\mathcal{P}(\tilde{\Sigma})$. The 't Hooft loop expectation value is then

$$
\langle\tilde{W}(\partial \tilde{\Sigma})\rangle \equiv \frac{\int \mathcal{D} U \exp \left(-\beta \sum_{U_{P} \in \mathcal{P}(\tilde{\Sigma})}\left(1-\frac{1}{N} \operatorname{Re} \operatorname{Tr} \zeta U_{P}\right)-\beta \sum_{U_{P} \notin \mathcal{P}(\tilde{\Sigma})}\left(1-\frac{1}{N} \operatorname{Re} \operatorname{Tr} U_{P}\right)\right)}{\int \mathcal{D} U \exp \left(-\beta \sum_{U_{P}}\left(1-\frac{1}{N} \operatorname{Re} \operatorname{Tr} U_{P}\right)\right)},
$$



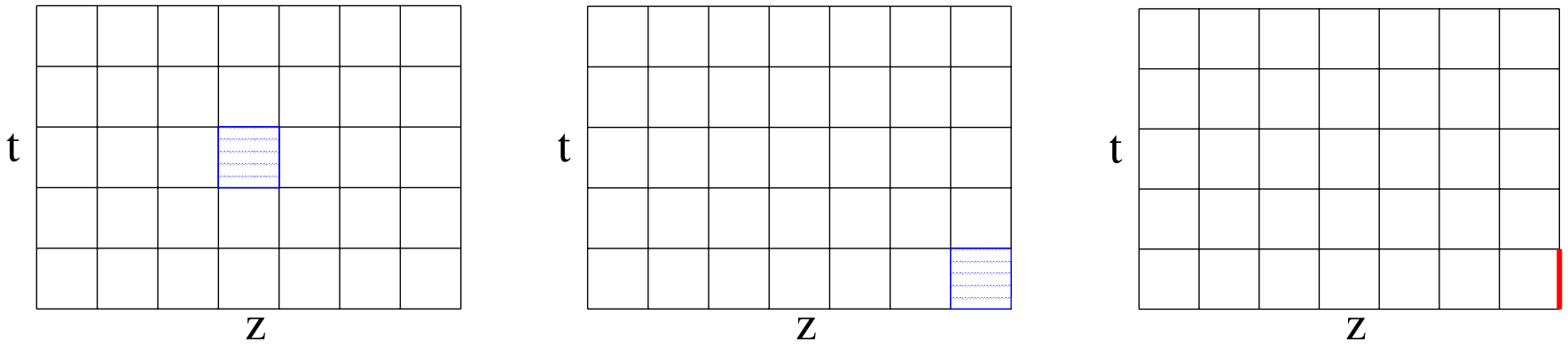

FIG. 1 (color online). How to create a 't Hooft loop $\partial \tilde{\Sigma}$ in the $(x, y)$ plane: (left) in each $(z, t)$ plane intersecting $\tilde{\Sigma}$, multiply by a center element one plaquette with coordinates $\left(z_{0}, t_{0}\right)$; (middle) equivalently, choose $\left(z_{0}, t_{0}\right)$ in the corner; (right) equivalently, multiply by a center element the link $U_{t}$ at the boundary. Thus, a 't Hooft loop of maximal size ( $\tilde{\Sigma}$ intersects all $(z, t)$ planes) is equivalent to twisted boundary conditions for the Polyakov loop.

where $\zeta \in Z_{N}$ (for $\mathrm{SU}$ (2), it amounts to flipping the coupling $\beta \rightarrow-\beta)$. Another choice of surface $\tilde{\Sigma}$ leaves $\langle\tilde{W}(\partial \tilde{\Sigma})\rangle$ invariant, as can be shown by a change of variables in (1.1): only the contour $\partial \tilde{\Sigma}$ matters. The dual string tension is then defined by

$$
\tilde{\sigma} \equiv \lim _{A(\partial \tilde{\Sigma}) \rightarrow \infty}-\frac{1}{A} \log \langle\tilde{W}(\partial \tilde{\Sigma})\rangle
$$

where $A$ is the minimal area bounded by $\partial \tilde{\Sigma}$. We can then consider a 't Hooft loop of maximum size $L_{x} \times L_{y}$, equal to that of the lattice in the $x$ and $y$ directions. In that case, the stack $\mathcal{P}(\tilde{\Sigma})$ contains one plaquette in every $(z, t)$ plane. Following Fig. 1 for each $(z, t)$ plane, we can move the special plaquette to a corner, then absorb the center element $\zeta$ into the boundary link $U_{t}{ }^{1}$ As a result, the timelike links satisfy $U_{t}\left(x, y, z+L_{z}, t=1\right)=$ $\zeta U_{t}(x, y, z, t=1)$. The same happens for the Polyakov loop $P(x, y, z) \equiv \prod_{t} U_{t}(x, y, z, t) \quad$ (the product of timelike links at position $(x, y, z))$, as

$$
P\left(x, y, z+L_{z}\right)=\zeta P(x, y, z) .
$$

Therefore, a 't Hooft loop of maximal size in the $(x, y)$ plane is equivalent to twisted boundary conditions for the Polyakov loop, and

$$
\left\langle\tilde{W}\left(L_{x}, L_{y}\right)\right\rangle=\frac{Z_{t b c}}{Z_{p b c}},
$$

where the numerator and denominator are the partition functions of a system with ordinary action, but with boundary conditions, respectively, twisted (by $\zeta$ ) and periodic in the $z$ direction for the Polyakov loop. Taking the logarithm on both sides, dividing by $L_{x} \times L_{y}$, and taking the thermodynamic limit, one recovers, on the left-hand side, the dual string tension $\tilde{\sigma}$, and on the right-hand

\footnotetext{
${ }^{1}$ The center element $\zeta$ could be absorbed in $U_{z}$ instead, leading to the same free energy for a system with twisted boundary conditions for the $z$-like "Polyakov" loop.
}

side, the interface free energy per unit 2dimensional area, i.e. the reduced interface tension, conventionally written as $\sigma / T$ in a rather confusing notation. The dual string tension is identical to the reduced interface tension. Therefore, all the old studies of $\mathrm{SU}(N)$ interface tensions, both numerical [7] and perturbative [8], can be relabeled as 't Hooft loop studies. It also becomes intuitively clear why $\tilde{\sigma}$ vanishes below $T_{c}$ : the Polyakov loop becomes disordered as the $Z(N)$ center symmetry is restored, and the interface tension, i.e. $\tilde{\sigma}$, vanishes.

(iii) Third, the numerical study of the 't Hooft loop has been considered more difficult than that of the Wilson loop. The reason is an "overlap" problem. In the 't Hooft loop expectation value Eq. (1.4), the two partition functions $Z_{t b c}$ and $Z_{p b c}$ are physically different. Gauge configurations which dominate the integral in the numerator $Z_{t b c}$ contain an interface; configurations which dominate in the denominator $Z_{p b c}$ do not. These two ensembles have little overlap, and importance sampling with respect to the denominator fails. This technical problem can be approached in various ways, all of which entail multiple simulations and increased computer cost. Decisive progress was achieved with the "snake" algorithm [6,9] which factorizes the ratio $Z_{t b c} / Z_{p b c}$ into $N_{x} \times N_{y}$ factors, each of $\mathcal{O}(1)$, which can each be estimated by an independent Monte Carlo simulation. In each factor, the area of the interface is increased by one elementary plaquette $a^{2}$. Finally, it was realized in [10] that a single factor converges to $\exp \left(-\tilde{\sigma} a^{2}\right)$ in the thermodynamic limit. This brings the computing cost of $\tilde{\sigma}$ on a par with that of the string tension $\sigma$ of the Wilson loop (actually, we will see that the force $\tilde{\sigma}$ between two dual charges can be computed with a constant accuracy independent of their separation, in sharp contrast with the ordinary string tension). Contact with perturbation theory for $\tilde{\sigma}(T)$ becomes feasible at high temperature, as we will show. 
One surprise comes on the way. We expect our lattice measurement of $\tilde{\sigma}(T)$ to be affected by discretization errors $\mathcal{O}\left(a^{2}\right)$, as for any bosonic theory, i.e.

$$
\tilde{\sigma}(T, a)=\tilde{\sigma}(T, a=0)\left(1+c_{1} a^{2}+\ldots\right),
$$

where $c_{1}$ starts with $\mathcal{O}\left(g^{2}\right)$. Here, $c_{1}$ starts with $\mathcal{O}\left(g^{0}\right)$, which can be traced to the nonperturbative nature of $\tilde{\sigma}$. In this paper, we calculate the complete correction $(\tilde{\sigma}(T, a) / \tilde{\sigma}(T, a=0)-1)$ for $g=$ 0 . It turns out to be large and quite different from $\mathcal{O}\left(a^{2}\right)$ for the lattice sizes $N_{t}$ accessible to current numerical simulations. In fact, it is not even monotonic as a function of $N_{t}$. The expected behavior $\sim c / N_{t}^{2}, c \ll 1$, is only recovered for $N_{t} \geqslant 8$. These large lattice corrections obscure the analysis of lattice data, and mar the continuum extrapolations of old SU(3) studies of the critical interface tension [7]. Our 1-loop calculation of these lattice correction factors, shown in Table I and Fig. 4, should be of general use.

Finally, we are in a position to compare our lattice Monte Carlo results for SU(2) with continuum perturbation theory $[2,3,8,11]$. Remarkable agreement is seen (cf. Figure 7), at the $2 \%$ level, down to temperatures $\mathcal{O}(10) T_{c}$, with perturbation theory at $\mathcal{O}\left(g^{2}\right)$.

Our paper is organized as follows. In Sec. II, we recall for completeness the perturbative calculation of the interface tension of Ref. [8], then show how this calculation is modified on the lattice, and extract the lattice correction factors Table I. In Sec. III, we compare our Monte Carlo results with the perturbative ones. Conclusions follow.

\section{1-LOOP CALCULATION OF THE INTERFACE TENSION}

\section{A. Continuum derivation}

Following [8], we calculate the $Z(2)$ interface tension, which is derived from the effective action of a kink interpolating between the two $S U(2)$ vacua. The effective action is the sum of the classical action plus a quantum term, obtained by integrating out fluctuations at 1-loop order. The calculation is done in euclidean space-time at temperature $T$. The euclidean time $x_{0}$ runs from 0 to $1 / T$. The system has spatial size $L_{x} \times L_{y} \times L_{z}$, which is taken to infinity in the end. A $Z(2)$ interface along the $z$-direction is constructed by assigning one vacuum to $z=0$ and the other to $z=L_{z}$, and minimizing the effective action subject to these boundary conditions. By definition, the interface tension $\tilde{\sigma}$ is equal to the action of this interface divided by the area of the plane $L_{x} L_{y}$ in the thermodynamic limit ( $\tilde{\sigma}$ is often called the "reduced" interface tension; one can equivalently consider the (full) interface tension $\tilde{\sigma}_{F}$, which is the interface action divided by the transverse volume $L_{x} L_{y} / T$. Of course, $\tilde{\sigma}=\tilde{\sigma}_{F} / T$ ). We consider a gauge field $A$ which is nontrivial only in the $x_{0}$-direction:

$$
\begin{gathered}
A_{\mu}^{c l}=C_{\mu} \tau_{3} ; \quad C_{0}=\frac{2 \pi T}{g} q, \quad C_{i}=0, \\
i=1,2,3,
\end{gathered}
$$

where $\tau_{3}$ is the diagonal generator of $\operatorname{SU}(2)\left(\tau_{3}=\sigma_{3} / 2\right)$. Then the Polyakov loop is

$$
P(\mathbf{x})=\frac{1}{2} \operatorname{Tr}\left[\mathcal{P} \exp \left(i g \int_{0}^{1 / T} d x_{0} A_{0}^{c l}(x)\right)\right]=\cos (\pi q) .
$$

The trivial vacuum is at $A_{\mu}^{c l}=q=0$ with $P(\mathbf{x})=1$. The $Z$ (2) transform of the trivial vacuum occurs for $q=1$, with $P(\mathbf{x})=\exp (i \pi)=-1$. We can therefore introduce an interface by choosing $q$ in (2.1) to be a function of $z$ and fixing $q(0)=0$ and $q\left(L_{z}\right)=1$. Then $P(\mathbf{x})=1$ at $z=0$ and $P(\mathbf{x})=-1$ at $z=L_{z}$. We need to justify why the path of minimal action between the two vacua can be brought to the form (2.1), with $q=q(z)$. That is because a constant background field can be brought to the diagonal, Cartan subalgebra by a global gauge rotation. But for $\mathrm{SU}(2)$, there is only one diagonal generator. The classical action is

$$
S^{c l}(A)=\int_{0}^{1 / T} d x_{0} \int d^{3} x \frac{1}{2} \operatorname{Tr}\left[G_{\mu \nu}^{2}\right],
$$

with the field-strength tensor $G_{\mu \nu}=\partial_{\mu} A_{\nu}-\partial_{\nu} A_{\mu}-$ $i g\left[A_{\mu}, A_{\nu}\right]$. For the field in (2.1) the classical action is

$$
S^{c l}=\frac{2}{g^{2}} T L_{x} L_{y} \pi^{2} \int_{0}^{L_{z}} d z\left[\frac{d q(z)}{d z}\right]^{2} .
$$

Minimizing the action subject to the boundary conditions $q(0)=0, q\left(L_{z}\right)=1$, yields $q(z)=z / L_{z}$. There is no true interface since the action vanishes like $1 / L_{z}$ when $L \rightarrow \infty$ is taken. But this is to be expected: classically there is no energy barrier, since the action is constant for any value of $q$. This degeneracy of the action with respect to $q$ is broken by quantum effects. To show this, we calculate the action in the presence of the (classical) background field defined in (2.1). To do this, we first assume $q$ to be constant in spacetime. The neglect of gradient terms will be justified $a$ posteriori. With $A_{\mu}=A_{\mu}^{c l}+A_{\mu}^{q u}$ the Lagrangian consists of the following Yang-Mills term plus gauge fixing term (background field gauge) and Faddeev-Popov ghost term

$$
\begin{gathered}
\mathcal{L}=\mathcal{L}_{\mathrm{YM}}+\mathcal{L}_{\mathrm{GF}}+\mathcal{L}_{\mathrm{FPG}} \quad \mathcal{L}_{\mathrm{YM}}=\frac{1}{2} \operatorname{Tr}\left[G_{\mu \nu}^{2}\right] \\
\mathcal{L}_{\mathrm{GF}}=\frac{1}{\xi} \operatorname{Tr}\left[F^{2}\right] \quad \mathcal{L}_{\mathrm{FPG}}=\operatorname{Tr}[\bar{\eta} P \eta] .
\end{gathered}
$$

where the gauge fixing term is $F=D_{\mu}^{c l} A_{\mu}^{q u}$. The operator $P$ in the ghost part is defined as $P \equiv-2 g\left(\delta_{\Lambda} F / \delta \Lambda\right)$, with a gauge transformation $\Lambda$. Also we define the covariant derivative in the adjoint representation for the background 
field:

$$
D_{\mu}^{c l}=\partial_{\mu}-i g\left[A_{\mu}^{c l},\right] .
$$

The whole Lagrangian quadratic in the fields is:

$$
\begin{aligned}
\mathcal{L}_{\mathrm{YM}}+\mathcal{L}_{\mathrm{GF}}= & \operatorname{Tr}\left[A _ { \mu } ^ { q u } ( x ) \left(-\delta_{\mu \nu} D_{c l}^{2}\right.\right. \\
& \left.\left.+\left(1-\frac{1}{\xi}\right) D_{\mu}^{c l} D_{\nu}^{c l}\right) A_{\nu}^{q u}(y)\right] \delta^{4}(x-y) \\
\mathcal{L}_{\mathrm{FPG}}= & \operatorname{Tr}\left[\bar{\eta}(x)\left(-2 D_{c l}^{2}\right) \eta(y)\right] \delta^{4}(x-y) .
\end{aligned}
$$

Using Feynman gauge $(\xi=1)$ it follows:

$$
\begin{aligned}
\mathcal{L} & =\mathcal{L}_{\mathrm{YM}}+\mathcal{L}_{\mathrm{GF}}+\mathcal{L}_{\mathrm{FPG}} \\
& =\frac{1}{2} A_{\mu a}^{q u}(x) \mathcal{M}_{\mu \nu}^{a b}(x, y) A_{\nu b}^{q u}(y)+\bar{\eta}_{a}(x) \mathcal{F}_{a b}(x, y) \eta_{b}(y)
\end{aligned}
$$

$$
\begin{array}{r}
\mathcal{F}_{a b}(x, y)=\delta^{4}(x-y)\left[-D_{c l}^{2}\right]_{a b} \\
\mathcal{M}_{a b}^{\mu \nu}(x, y)=\delta_{\mu \nu} \delta^{4}(x-y)\left[-D_{c l}^{2}\right]_{a b} .
\end{array}
$$

To obtain the quantum effective action to one-loop $S_{1}^{q u}$ we have to integrate out the quantum fields $A^{q u}, \bar{\eta}, \eta$

$$
\begin{aligned}
e^{-S_{1}^{q u}} & =\int \mathcal{D} A^{q u} \mathcal{D} \bar{\eta} \mathcal{D} \eta e^{-S} \\
& =\int \mathcal{D} A^{q u} \mathcal{D} \bar{\eta} \mathcal{D} \eta \exp \left[-\frac{1}{2} \int d^{4} x d^{4} y A_{\mu a}^{q u}(x) \mathcal{M}_{\mu \nu}^{a b}(x, y) A_{\nu b}^{q u}(y)-\int d^{4} x d^{4} y \bar{\eta}_{a}(x) \mathcal{F}_{a b}(x, y) \eta_{b}(y)\right] \\
\Rightarrow S_{1}^{q u} & =\frac{1}{2} \operatorname{Tr}[\log (\mathcal{M})]-\operatorname{Tr}[\log (\mathcal{F})] .
\end{aligned}
$$

To calculate such traces it is helpful to diagonalize the "matrices" $\mathcal{M}$ and $\mathcal{F}$ by passing to momentum space. We then need the eigenvalues of the matrix

$$
-\tilde{D}_{c l}^{2}(k)=\left(\begin{array}{ccc}
\mathbf{k}^{2}+k_{0}^{2}+(2 \pi T q)^{2} & 2 i(2 \pi T q) k_{0} & 0 \\
-2 i(2 \pi T q) k_{0} & \mathbf{k}^{2}+k_{0}^{2}+(2 \pi T q)^{2} & 0 \\
0 & 0 & \mathbf{k}^{2}+k_{0}^{2}
\end{array}\right)
$$

At finite temperature, $k_{0}=2 \pi T n$ with $n$ integer, and, using the definition

$$
k_{ \pm} \equiv 2 \pi T(n \pm q),
$$

it follows

$$
\begin{aligned}
& \lambda_{1}=\mathbf{k}^{2}+[2 \pi T(n+q)]^{2}=\mathbf{k}^{2}+k_{+}^{2} \\
& \lambda_{2}=\mathbf{k}^{2}+[2 \pi T(n-q)]^{2}=\mathbf{k}^{2}+k_{-}^{2} \\
& \lambda_{3}=\mathbf{k}^{2}+k_{0}^{2} \quad \text { (independent of } q \text { ). }
\end{aligned}
$$

This shift in momenta is a typical effect of a constant background field. The sum over $k_{+}$is equal to the sum over $k_{-}$, therefore the quantum action reads, after substitution of (2.10) and (2.11) into (2.12):

$$
S_{1}^{q u}=V \sum_{n=-\infty}^{+\infty} \int \frac{d^{3} \mathbf{k}}{(2 \pi)^{3}} 2 \log \left(\mathbf{k}^{2}+k_{+}^{2}\right),
$$

where terms independent of $q$ (eigenvalue $\lambda_{3}$ ) have been dropped and the sum is over all Matsubara modes $n$. Now we need to perform the sums. To do this, we first differentiate with respect to $q$.

$$
\begin{aligned}
\frac{\partial S_{1}^{q u}}{\partial q} & =\frac{T V}{\pi^{2}} \sum_{n=-\infty}^{+\infty} \int d^{3} \mathbf{k} \frac{k_{+}}{\mathbf{k}^{2}+k_{+}^{2}} \\
& =-8 T^{3} V \pi^{2} \sum_{n=-\infty}^{+\infty}(n+q)|n+q| .
\end{aligned}
$$

The divergent sum is interpreted using zeta-function regularization, with $\zeta(s, q) \equiv \sum_{n=0}^{+\infty}(n+q)^{-s}$. Noting that $\sum_{n=-\infty}^{+\infty}(n+q)|n+q|=\zeta(-2, q)-\zeta(-2,1-q), \quad$ and using the identity $\zeta(s, q)=\left[B_{-s+1}(q)\right] /(s-1)$ for $s \leq$ 0 , where $B$ is the Bernouilli polynomial, one gets $\left(d S_{1}^{q u} / d q\right)=\frac{4}{3} T^{3} V \pi^{2}(d / d q)\left[q^{2}(1-q)^{2}\right] . \quad$ Integrating back with respect to $q$, one obtains:

$$
S_{1}^{q u}=\frac{4}{3} T^{3} V \pi^{2}\left[q^{2}(1-q)^{2}\right] .
$$

So far we treated $q$ as if constant, but actually we want it to be a function $q=q(z)$ of $z$. The reason why we can still make use of the action $S_{1}^{q u}$ for constant $q$ is, that $A^{c l}$ varies slowly with $z$, and at leading order this variation can be neglected (gradient expansion), as we will see in the final kink solution (2.21). Now we reintroduce the $z$-dependence of $q \rightarrow q(z)$ and replace the size of the system in the $z$ direction $L_{z}\left(V=L_{x} L_{y} L_{z}\right)$ by an integral $L_{z} \rightarrow \int_{0}^{L_{z}} d z$. 


$$
S_{1}^{q u}=\frac{4}{3} T^{3} L_{x} L_{y} \pi^{2} \int_{0}^{L_{z}} d z\left[q(z)^{2}(1-q(z))^{2}\right] .
$$

Now the classical degeneracy in $q$ has been lifted by quantum effects and the minima (vacua) are at $q=0$ and $q=1$ (see Fig. 2, left). Since the sum (2.17) is periodic in $q$ and hence invariant under shifts $q \rightarrow q+k$ for any integer $k$, this is also true for the quantum action. Thus $q$ in $S_{1}^{q u}$ (2.19) is defined modulo one. From (2.4) the classical action is

$$
S^{c l}=\frac{2}{g^{2}} T L_{x} L_{y} \pi^{2} \int_{0}^{L_{z}} d z\left[\frac{d q(z)}{d z}\right]^{2} .
$$

Now introduce the dimensionless coordinate $z^{\prime}=c(z-$ $\left.L_{z} / 2\right)$ with $c=\sqrt{\frac{2}{3}} g T$ in both $S^{c l}$ and $S_{1}^{q u}$. The complete effective kink action then becomes:

$$
\begin{aligned}
S^{\mathrm{kink}} & =S^{c l}+S_{1}^{q u} \\
& =\sqrt{\frac{2}{3} \frac{2}{g}} T^{2} L_{x} L_{y} \pi^{2} \int_{-L_{z}^{\prime}}^{L_{z}^{\prime}} d z^{\prime}\left[\left(\frac{d q}{d z^{\prime}}\right)^{2}+q^{2}(1-q)^{2}\right],
\end{aligned}
$$

with $L_{z}^{\prime}=c L_{z} / 2$. Defining the Lagrangian $\mathcal{L}=$ $\left(d q / d z^{\prime}\right)^{2}+V(q), \quad V(q) \equiv q^{2}(1-q)^{2}, \quad$ the EulerLagrange equation of motion gives $d q / d z^{\prime}=\sqrt{V(q)}$. Taking $L_{z} \rightarrow \infty$ the solution which satisfies the boundary conditions $q(0)=0$ and $q\left(L_{z}\right)=1$ is

$$
q(z)=\frac{e^{z^{\prime}}}{1+e^{z^{\prime}}}, z^{\prime}=c\left(z-\frac{L_{z}}{2}\right) .
$$

This solution is called a kink (see Fig. 2, right). The width of the kink is $c^{-1} \sim 1 / g T$, which is much larger than the scale $1 / T$ of our system since $g \ll 1$. Hence it was indeed justified to neglect gradient terms $d q / d z$ : they only contribute at the next order in $g^{2}$. Using the equation of motion, the integral in (2.20) becomes $2 \int_{0}^{1} d q \sqrt{V(q)}$.

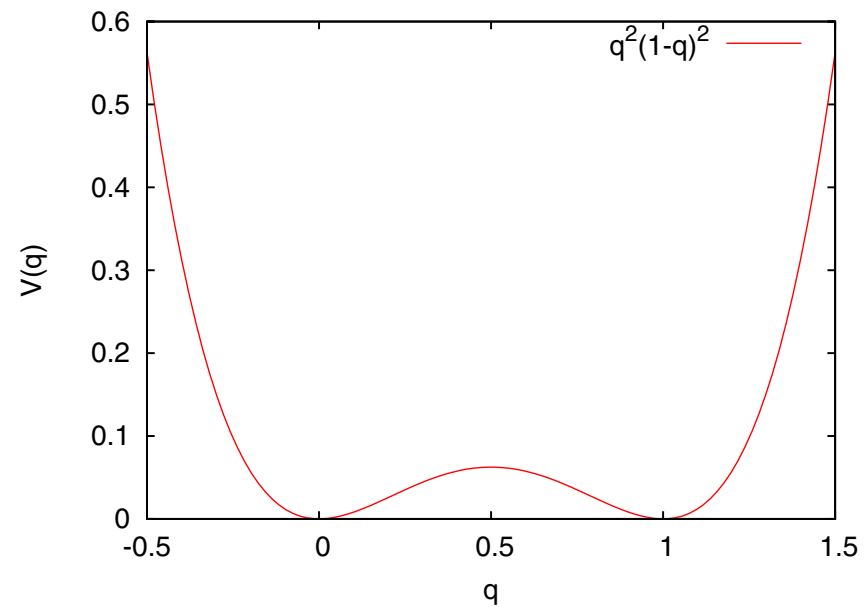

Performing the integration, we finally obtain for the effective action of the kink between the two vacua

$$
S^{\mathrm{kink}}=\frac{4 \pi^{2}}{3 \sqrt{6}} \frac{T^{2}}{g} L_{x} L_{y} .
$$

Our interface tension $\tilde{\sigma}$ is the kink action per unit planar area, i.e. $S^{\mathrm{kink}} / L_{x} L_{y}$ :

$$
\tilde{\sigma}(T)=\frac{4 \pi^{2}}{3 \sqrt{6}} \frac{T^{2}}{g} .
$$

This formula superficially differs from the corresponding one in [8] by a factor $T$, but this is due to our different definition of the interface tension $\left(S^{\mathrm{kink}}=\left(L_{x} L_{y} / T\right) \tilde{\sigma}_{F}\right.$ in [8]).

\section{B. Corrections on the lattice}

When measuring the interface tension on the lattice, one may expect that the measurements of $\tilde{\sigma} / T^{2}\left(N_{t}\right)\left(N_{t}\right.$ number of temporal sites) approach the perturbative continuum form (2.23) for $g \rightarrow 0$. Somewhat surprisingly, this is not the case. Multiplicative correction factors are needed, which are large for all $N_{t}$ accessible to numerical simulations. Only for very large $N_{t}$ do these factors go to 1 . In [12], Nathan Weiss gave the main expression leading to these correction factors, but without giving details about the calculation. This is done here for completeness. Moreover, we show how well these results describe the measurements we obtain from Monte Carlo simulations.

Our derivation on the lattice is very similar to the one in the continuum and in the end, we compare the obtained effective potentials from both calculations. Actually the only thing to do is to find a lattice expression for the inverse propagator $D_{c l}^{2}$, which took the form (2.13) in the continuum.

A four-dimensional lattice of size $a N_{s}$ in the spatial directions and $a N_{t}$ in the temporal direction is used. We

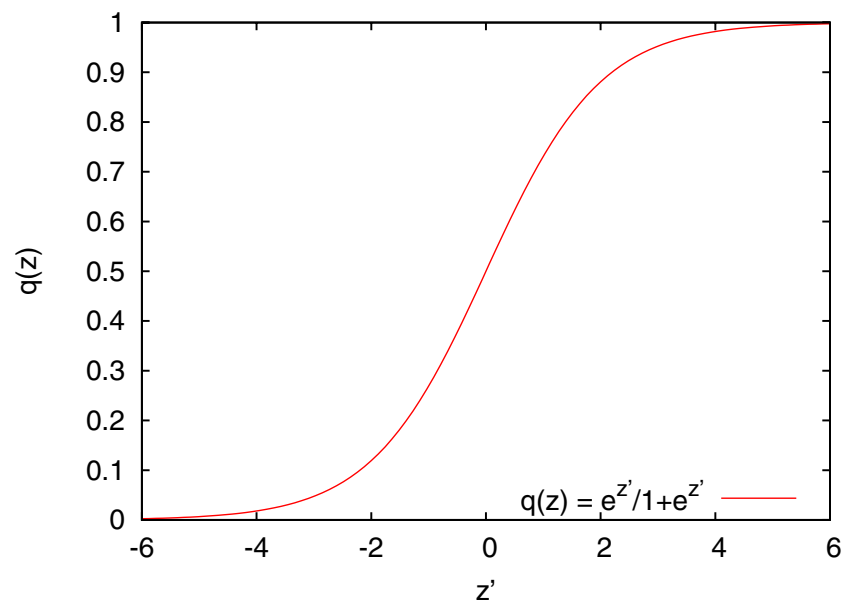

FIG. 2 (color online). Left: The 1-loop effective potential is the function shown in the interval $[0,1]$, then periodically repeated. Right: the kink solution for the effective potential, as a function of $z^{\prime}=\sqrt{\frac{2}{3}} g T z$. 
calculate the classical action first. The Wilson action is

$$
S_{c l}^{L}=\beta \sum_{P}\left(1-\frac{1}{2} \operatorname{Re} \operatorname{Tr} U_{P}\right) .
$$

Only plaquettes in the $z t$ plane are different from $\mathbf{1}$. Such a plaquette at position $z$ takes the value

$$
U_{P}=e^{i a\left(\pi / N_{t}\right)(1 / a)(q(z+a)-q(z)) \sigma_{3}}=e^{i a\left(\pi / N_{t}\right) \Delta_{z}^{f} q(z) \sigma_{3}}
$$

with the forward derivative $\Delta_{z}^{f}$. The trace of the plaquette is

$$
2 \cos \left(a \frac{\pi}{N_{t}} \Delta_{z}^{f} q(z)\right) \approx 2\left(1-\frac{1}{2}\left(a \frac{\pi}{N_{t}} \Delta_{z}^{f} q(z)\right)^{2}\right) .
$$

$$
\left[-\tilde{D}_{c l}^{2 L}\right]=\left(\begin{array}{c}
\sum_{\mu} 4 \sin ^{2}\left(\frac{k_{\mu}^{\prime}}{2}\right)+4 \sin ^{2}\left(\frac{\pi q}{N_{t}}\right) \cos \left(k_{0}^{\prime}\right) \\
-2 i \sin \left(\frac{2 \pi q}{N_{t}}\right) \sin \left(k_{0}^{\prime}\right) \\
0
\end{array}\right.
$$

The eigenvalues are

$$
\begin{aligned}
& \lambda_{1}=\frac{4}{a^{2}}\left(\sum_{i=1}^{3} \sin ^{2}\left(\frac{k_{i}^{\prime}}{2}\right)+\sin ^{2}\left(\pi \frac{(n+q)}{N_{t}}\right)\right) \\
& \lambda_{2}=\frac{4}{a^{2}}\left(\sum_{i=1}^{3} \sin ^{2}\left(\frac{k_{i}^{\prime}}{2}\right)+\sin ^{2}\left(\pi \frac{(n-q)}{N_{t}}\right)\right) \\
& \lambda_{3}=\frac{4}{a^{2}}\left(\sum_{i=1}^{3} \sin ^{2}\left(\frac{k_{i}^{\prime}}{2}\right)+\sin ^{2}\left(\pi \frac{n}{N_{t}}\right)\right),
\end{aligned}
$$

which can be directly obtained from (2.15) by replacing momenta with their lattice counterparts $k \rightarrow \hat{k}=\frac{2}{a} \times$ $\sin \left(\frac{a}{2} k\right)$. The lattice quantum action is (dropping again the eigenvalue $\lambda_{3}$ since it is independent of $q$ ):

$$
\begin{aligned}
S_{1}^{q u L}= & V \sum_{n=0}^{N_{t}-1} \int_{0}^{2 \pi / a} \frac{d^{3} \mathbf{k}^{\prime}}{(2 \pi)^{3}}\left\{\operatorname { l o g } \left[\sum_{i=1}^{3} \sin ^{2}\left(\frac{k_{i}^{\prime}}{2}\right)\right.\right. \\
& \left.+\sin ^{2}\left(\pi \frac{(n+q)}{N_{t}}\right)\right]+\log \left[\sum_{i=1}^{3} \sin ^{2}\left(\frac{k_{i}^{\prime}}{2}\right)\right. \\
& \left.\left.+\sin ^{2}\left(\pi \frac{(n-q)}{N_{t}}\right)\right]\right\} .
\end{aligned}
$$

After performing the sum, we get, up to terms independent of $q$

$$
\begin{aligned}
S_{1}^{q u L}= & 2 N_{s}^{3} \int_{0}^{2 \pi} \frac{d^{3} \mathbf{k}^{\prime}}{(2 \pi)^{3}} \log \left[1-2 \cos (2 \pi q) e^{-2 N_{t} h}\right. \\
& \left.+e^{-4 N_{t} h}\right]
\end{aligned}
$$

Placing this into the action we have

$$
S_{c l}^{L}=\beta \sum_{P_{z t}} \frac{1}{2}\left(a \frac{\pi}{N_{t}} \Delta_{z}^{f} q(z)\right)^{2}=\frac{2}{g^{2}} \frac{N_{s}^{2}}{N_{t}} \pi^{2} a^{2} \sum_{z}\left(\Delta_{z}^{f} q(z)\right)^{2},
$$

where $\sum_{P_{z t}}$ means sum over plaquettes in the $z t$ plane and $\beta=4 / g^{2}$. Mimicking the steps from the continuum derivation, we add quantum fluctuations to this action. The calculation on the lattice yields for the momentum space propagator (2.13) $\left(k \rightarrow k^{\prime} / a\right.$ where $k^{\prime}$ is now a dimensionless quantity)

$$
\left.\begin{array}{cc}
2 i \sin \left(\frac{2 \pi q}{N_{t}}\right) \sin \left(k_{0}^{\prime}\right) & 0 \\
\sum_{\mu} 4 \sin ^{2}\left(\frac{k_{\mu}^{\prime}}{2}\right)+4 \sin ^{2}\left(\frac{\pi q}{N_{t}}\right) \cos \left(k_{0}^{\prime}\right) & 0 \\
0 & \sum_{\mu} 4 \sin \left(\frac{k_{\mu}^{\prime}}{2}\right)
\end{array}\right) .
$$

$$
h=\log \left(K+\sqrt{K^{2}+1}\right)
$$

$$
K^{2}=\sin ^{2}\left(\frac{k_{x}^{\prime}}{2}\right)+\sin ^{2}\left(\frac{k_{y}^{\prime}}{2}\right)+\sin ^{2}\left(\frac{k_{z}^{\prime}}{2}\right),
$$

which is the same as stated by Weiss in [12]. To obtain this result, we differentiated with respect to $h$, then used the identity

$$
\sum_{n=0}^{N_{t}-1} \frac{2 \sinh (h) \cosh (h)}{\sin ^{2}\left(\pi \frac{(n-q)}{N_{t}}\right)+\sinh ^{2}(h)}=\frac{N_{t} \sinh \left(2 N_{t} h\right)}{\sin ^{2}(\pi q)+\sinh ^{2}\left(N_{t} h\right)},
$$

noting that $K=\sinh (h)$. We finally integrated back to get (2.30). We want to compare this to the continuum expression to make a quantitative prediction for corrections to the interface tension measured on the lattice. From (2.18) we have in the continuum $\left(V=L_{x} L_{y} L_{z}\right)$

$$
S_{1}^{q u}=\frac{4}{3} V T^{3} \pi^{2}\left[q^{2}(1-q)^{2}\right] .
$$

Defining

$$
\begin{aligned}
V_{L}\left(q, N_{t}\right)= & \left(\frac{3}{2 \pi^{2}} N_{t}^{3}\right) \int_{0}^{2 \pi} \frac{d^{3} \mathbf{k}^{\prime}}{(2 \pi)^{3}} \\
& \times \log \left[1-2 \cos (2 \pi q) e^{-2 N_{t} h}+e^{-4 N_{t} h}\right],
\end{aligned}
$$

and subtracting $V_{L}(q=0)$ from the action in order to make it zero when the system is in one of the vacua we get on the lattice 


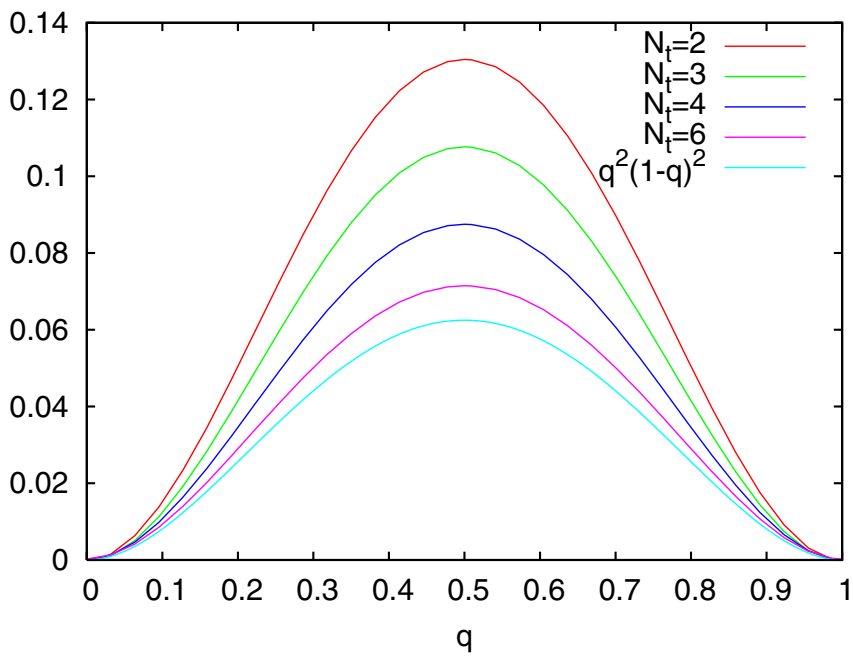

FIG. 3 (color online). The lattice effective potential $V_{L}\left(q, N_{t}\right)-V_{L}\left(0, N_{t}\right)$ for different $N_{t}$ 's, compared to the continuum effective potential $q^{2}(1-q)^{2}$.

$$
S_{1}^{q u L}=\frac{4}{3} V T^{3} \pi^{2}\left(V_{L}\left(q, N_{t}\right)-V_{L}\left(0, N_{t}\right)\right) .
$$

Comparing this to (2.34), we see that $V(q)=q^{2}(1-q)^{2}$ in the continuum is replaced by $V_{L}\left(q, N_{t}\right)-V_{L}\left(0, N_{t}\right)$ on the lattice. Both are shown in Fig. 3. If the continuum limit $N_{t} \rightarrow \infty$ is taken, we have $h \rightarrow \frac{|k|}{2}$ and therefore

$$
\begin{aligned}
V_{L}(q)= & \left(\frac{3}{2 \pi^{2}} N_{t}^{3}\right) \int_{0}^{\infty} \frac{4 \pi k^{2} d k}{(2 \pi)^{3}} \\
& \times \log \left[1-2 \cos (2 \pi q) e^{-N_{t} k}+e^{-2 N_{t} k}\right] \\
= & \left(\frac{3}{4 \pi^{4}}\right) \int_{0}^{\infty} x^{2} d x \log \left[1-2 \cos (2 \pi q) e^{-x}+e^{-2 x}\right],
\end{aligned}
$$

which can be shown to be identical to $q^{2}(1-q)^{2}+$ constant, by differentiating with respect to $q$. Mimicking the steps that led to (2.22), the corrections on the lattice are calculated by comparing

$$
\int_{0}^{1} d q \sqrt{V_{L}\left(q, N_{t}\right)-V_{L}\left(0, N_{t}\right)}
$$

with its continuum counterpart

$$
\int_{0}^{1} d q \sqrt{q^{2}(1-q)^{2}}=\frac{1}{6} .
$$

This gives for the lattice correction factors $C_{l a t}\left(N_{t}\right) \equiv$ $\tilde{\sigma}\left(N_{t}\right) / \tilde{\sigma}\left(N_{t}=\infty\right)$ :

$$
C_{\text {lat }}\left(N_{t}\right)=6 \int_{0}^{1} d q \sqrt{V_{L}\left(q, N_{t}\right)-V_{L}\left(0, N_{t}\right)}
$$

A numerical evaluation yields the factors in Table I. As is to be expected in a bosonic theory, the corrections are of order $O\left(a^{2}\right)$, i.e. the factors are of the form $\left(1+O\left(1 / N_{t}^{2}\right)\right)$.
TABLE I. Lattice correction factors $C_{l a t}$ versus the number $N_{t}$ of temporal slices.

\begin{tabular}{ll}
\hline \hline$N_{t}$ & $C_{\text {lat }}$ \\
\hline 1 & 1.2237 \\
2 & 1.4233 \\
3 & 1.29467 \\
4 & 1.17025 \\
5 & 1.09927 \\
6 & 1.06278 \\
7 & 1.04324 \\
8 & 1.03181 \\
9 & 1.02451 \\
10 & 1.01953 \\
\hline \hline
\end{tabular}

This is shown in Fig. 4. However, this asymptotic behavior sets in only for large values of $N_{t}$. For small values of $N_{t}$, the ones accessible to numerical simulations, the correction factors show a quite different, not even monotonic $\left(N_{t}=1\right)$, behavior. This knowledge is crucial when trying to extrapolate to the continuum limit with only small- $N_{t}$ Monte Carlo data. To leading order the lattice correction factors $C_{l a t}$ do not depend on the number of colors $N$, which only occurs as a prefactor in the 1-loop quantum action and cancels out when comparing the continuum to the lattice effective potential. Thus Table I applies to $\mathrm{SU}(N)$ as well. There is one more thing to say, concerning finite-size effects. So far, we chose the spatial dimensions $N_{s}$ to be $\infty$. But we can also calculate the impact of a finite size in the spatial dimensions on the lattice correction factors. Let us consider a cubic geometry for simplicity. For finite $N_{s}$ the integral over the momenta $\mathbf{k}$ becomes a sum and we obtain

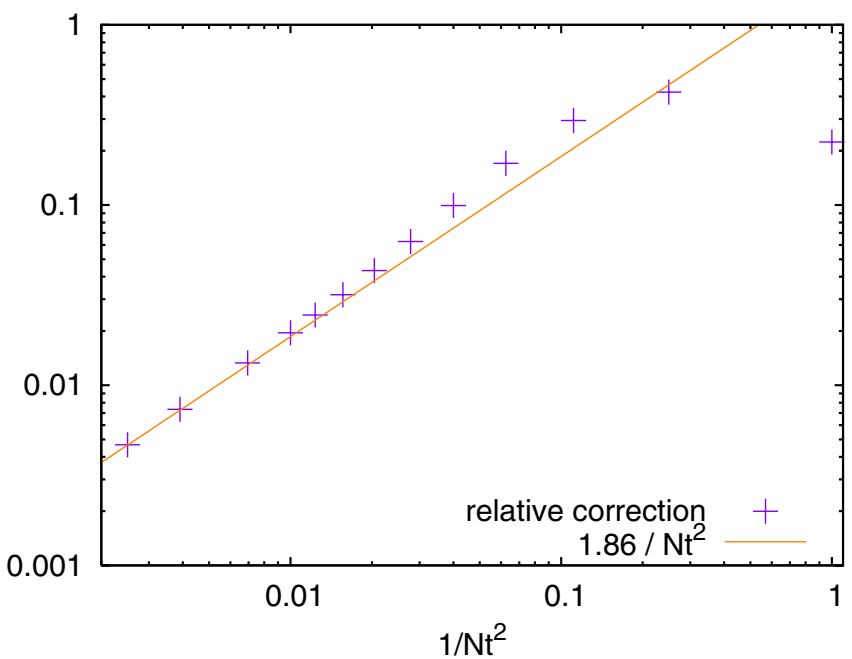

FIG. 4 (color online). The relative correction to the interface tension $\tilde{\sigma},\left(C_{\text {lat }}\left(N_{t}\right)-1\right)$, for different values of $N_{t}$. Only for large values of $N_{t}$ does the relative correction show the expected behavior $\propto 1 / N_{t}^{2}$. 


$$
\begin{aligned}
V_{L}\left(q, N_{t}, N_{s}\right)= & \frac{3}{2 \pi^{2}}\left(\frac{N_{t}}{N_{s}}\right)^{3} \\
& \times \sum_{n_{x}, n_{y}, n_{z}=0}^{N_{s}-1} \log \left[1-2 \cos (2 \pi q) e^{-2 N_{t} h}\right. \\
& \left.+e^{-4 N_{t} h}\right] \\
K^{2}= & \sin ^{2}\left(\frac{\pi}{N_{s}} n_{x}\right)+\sin ^{2}\left(\frac{\pi}{N_{s}} n_{y}\right)+\sin ^{2}\left(\frac{\pi}{N_{s}} n_{z}\right) .
\end{aligned}
$$

One finds, for example, at $N_{t}=2$ and aspect ratio $\rho=2$ $\left(\rho \equiv N_{s} / N_{t}\right)$ that $C_{\text {lat }}$ in Table I should be multiplied by 0.885, for $\rho=4: 0.966$ and for $\rho=8: 0.992$. This shows, that with increasing aspect ratio, the impact of a finite $N_{s}$ becomes quickly negligible. If finite-size effects are taken into account, $C_{\text {lat }}$ decreases slightly, and therefore the corrected interface tension $\tilde{\sigma}(T) / C_{\text {lat }}$ increases by a tiny amount.

\section{COMPARING WITH SIMULATIONS}

\section{A. Measuring the interface tension}

A direct Monte Carlo measurement of $\tilde{\sigma}$ from the free energy definition (1.4),(1.2) is impractical. First, there is an overlap problem: configurations contributing to the numerator and the denominator of (1.4) are physically, macroscopically different, since they, respectively, contain an interface or do not. Thus, importance sampling with respect to the denominator fails for all but the smallest volumes. Second, the interface is translationally invariant, so that the corresponding entropy must be carefully subtracted to avoid $\log L$ corrections. Both problems can be solved by a clever choice of algorithm. Historically, this choice has been arrived at in two steps.

An elegant approach to the overlap problem is provided by the "snake" algorithm [6,9]. It consists of building the interface in $N_{x} \times N_{y}$ steps, one plaquette at a time, and factorizing the ratio Eq. (1.4):

$$
\frac{Z_{t b c}}{Z_{p b c}}=\frac{Z_{N}}{Z_{N-1}} \cdot \frac{Z_{N-1}}{Z_{N-2}} \cdot \ldots \cdot \frac{Z_{1}}{Z_{0}},
$$

where $N=N_{x} \times N_{y}$, and $Z_{k}, k=0, \ldots, N\left(Z_{N} \equiv Z_{t b c}\right.$ and $Z_{0} \equiv Z_{p b c}$ ) is the partition function for a 't Hooft loop of area $k a^{2}$, with a stack $\mathcal{P}_{k}$ of $k$ corresponding plaquettes "twisted", i.e. having the coupling $\beta$ changed to $-\beta$ :

$$
\begin{aligned}
Z_{k}= & \int \mathcal{D} U \exp \left(+\beta \sum_{\mathcal{P}_{k}}\left(1-\frac{1}{2} \operatorname{Re} \operatorname{Tr} U_{P}\right)\right. \\
& \left.-\beta \sum_{\overline{\mathcal{P}}_{k}}\left(1-\frac{1}{2} \operatorname{Re} \operatorname{Tr} U_{P}\right)\right),
\end{aligned}
$$

where $\overline{\mathcal{P}}_{k}$ is the complement of $\mathcal{P}_{k}$. When $\bmod \left(k, N_{y}\right)=0$, the 't Hooft loop is rectangular with perimeter $2 N_{y}$, otherwise it has perimeter $\left(2 N_{y}+2\right)$, and one side contains 2 kinks (the bold $x$ links in Fig. 5). The progressive flipping of the plaquettes is described by a snakelike motion, hence the name attached to the algorithm [9]. Each ratio in (3.1) is of $\mathcal{O}(1)$ and can be efficiently estimated by a separate Monte Carlo simulation.

In fact, this algorithm can be simplified [10], and the $N_{x} \times N_{y}$ Monte Carlo simulations reduced to a single one, by the following observation. $Z_{k+1} / Z_{k}=$ $\left(Z_{k+1} / Z_{0}\right) /\left(Z_{k} / Z_{0}\right)$ is a ratio of two 't Hooft loops differing by $a^{2}$ in area. If $\bmod \left(k, N_{y}\right) \neq\{0,-1\}$, they have the same perimeter and number of corners. They form the dual analogue of a Creutz ratio, commonly used to measure the ordinary string tension. Therefore,

$$
\lim _{N_{x}, N_{y} \rightarrow \infty}-\log \frac{Z_{k+1}}{Z_{k}}=\tilde{\sigma} a^{2} .
$$

A crucial advantage over the Creutz ratio is that the integrand in $Z_{k}$ is of exponential form: it is positive and can be used as a sampling probability. Then, a given statistical precision on $Z_{k+1} / Z_{k}$ requires the same computer effort, independently of the 't Hooft loop size. As stressed in [13], this is in sharp contrast to the ordinary string tension, where the statistical accuracy degrades exponentially with the Wilson loop area. Therefore, (3.3) can be rewritten and measured as an expectation value with respect to $Z_{k}$ :

$$
\tilde{\sigma} a^{2}=\lim _{N_{x}, N_{y} \rightarrow \infty}-\log \left\langle\exp \left(-2 \beta \frac{1}{2} \operatorname{Re} \operatorname{Tr} U_{P_{k+1}}\right)\right\rangle .
$$

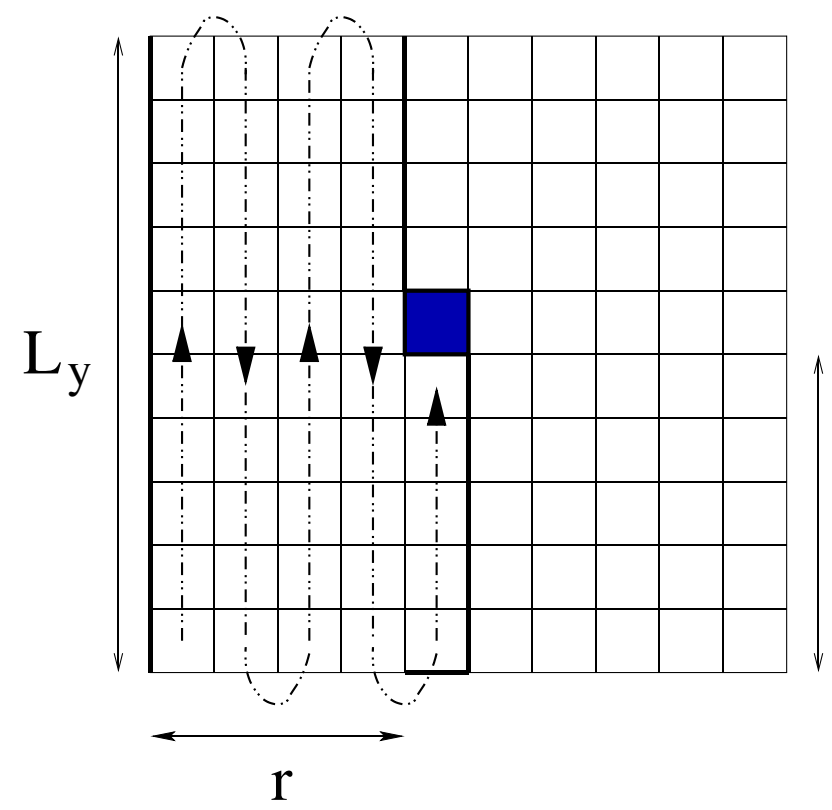

FIG. 5 (color online). Illustration of the snake algorithm, which builds the interface one plaquette at a time, and of its simplified version, which requires a single Monte Carlo simulation of a system with a partial interface. 
In our simulations, we use the equivalent $\left\langle\exp \left(-\beta \frac{1}{2} \operatorname{Re} \operatorname{Tr} U_{P_{k+1}}\right)\right\rangle_{0} /\left\langle\exp \left(+\beta \frac{1}{2} \operatorname{Re} \operatorname{Tr} U_{P_{k+1}}\right)\right\rangle_{0}$, where the expectation value $\langle. .\rangle_{0}$ is taken in the ensemble which interpolates between $Z_{k}$ and $Z_{k+1}$, where the coupling attached to $U_{P_{k+1}}$ is set to zero. This observable has a smaller variance, which can be further reduced by performing multihit (analytically) over the 4 links making up $U_{P_{k+1}}$, since they are now decoupled from each other.

Note that translation invariance is broken. Our 't Hooft loops $Z_{k}$ and $Z_{k+1}$ have fixed boundaries, and the observable is a function of a single plaquette in the system. This solves the second problem mentioned at the beginning: there is no entropy to subtract from the free energy of a partial interface. ${ }^{2}$ It also suggests to organize the Monte Carlo updates hierarchically in shells centered around $P_{k+1}$ [13]. The gauge links of the innermost shells, upon which the observable depends most sensitively, are integrated more thoroughly with more frequent Monte Carlo updates.

Since taking a large 't Hooft loop in (3.4) requires no more work than taking a small one, we choose the size as large as practical, as per Fig. 5, with length $N_{y}$ and width $r \sim N_{x} / 2$. $r$ can be increased even more, but taking it too close to $N_{x}$ brings about the possibility of reducing the total free energy by trading the single interface of width $r$ for two of them: one interface of width $\left(N_{x}-r\right)$, and one full interface of width $N_{x}$. Translation invariance of the latter causes the free energy reduction. The choice $r \sim N_{x} / 2$ completely eliminates this finite-size effect.

For large 't Hooft loops of size $N_{y}, r \gg \xi=1 / \sqrt{\tilde{\sigma} a^{2}}$, the leading deviation of (3.4) from its asymptotic value comes from two contributions: Gaussian fluctuations of the interface away from its minimal area (known as capillary waves, or Lüscher correction), and interaction between the two kinks in the 't Hooft loop perimeter Fig. $5 .^{3}(i)$ The Lüscher correction can be evaluated analytically. For a vibrating $L_{y} \times r$ interface with $\xi \ll r \ll L_{y}$, it takes the familiar form $\tilde{\sigma} \rightarrow \tilde{\sigma}+\frac{\pi}{12} \frac{1}{r^{2}}$. In our case where $r \sim \frac{1}{2} L_{y}$, it is obtained from the partition function appropriate to our boundary conditions [14]

$$
Z_{\text {surface }}=\eta^{2}\left[i \frac{L_{y}}{2 r}\right], \quad \eta(\tau)=e^{i(\pi / 12) \tau} \prod_{n=1}^{\infty}\left(1-e^{i 2 \pi n \tau}\right),
$$

We subtract this analytic correction from our Monte Carlo results. (ii) A kink of size $a$ in the 't Hooft loop perimeter

\footnotetext{
${ }^{2}$ In the original snake algorithm, translation invariance is restored in the last ratio $Z_{N} / Z_{N-1}$. Its Monte Carlo evaluation is plagued by very long autocorrelation times, corresponding to translations of the full interface.

${ }^{3}$ Excited states of the interface also bring a correction. Assuming an energy gap $\pi / r$ between the ground and first excited state propagating along the $y$ direction, one can check that this correction is smaller than our statistical errors, for all lattice sizes and couplings considered here.
}

creates UV excited string states of energy $\mathcal{O}\left(a^{-1}\right)$, which propagate along the $y$ direction over a distance $s \approx a N_{y} / 2$. Therefore, we expect a correction of order $\exp \left(-N_{y} / 2\right)$ due to the two kinks. Indeed, Monte Carlo data obtained on lattices of increasing size $L^{3} \times 2$ at fixed $\beta$ are well described by the ansatz $\left(\tilde{\sigma} a^{2}+\right.$ Lüscher correction $\left.+c_{1} \exp \left(-c_{2} N_{y} / 2\right)\right)$ with $c_{1}, c_{2} \sim 1$ [10]. This finite-size correction is negligible compared to our statistical errors provided $N_{y} \gg-2 \log \left(\tilde{\sigma} a^{2}\right)$. Finally, numerical tests at several values of $\beta$ and several lattice extents $N_{t}$ indicated that finite-size effects cause an underestimate of the true interface tension, and led us to aspect ratios $\left\{N_{x}, N_{y}, N_{z}\right\} / N_{t}$ as large as 16 .

To increase statistics, we typically estimated 2 ratios $Z_{k+1} / Z_{k}$, corresponding to plaquettes $U_{P_{k+1}}$ at the "center" of the lattice (i.e. with coordinates $\left(N_{x} / 2, N_{y} / 2+\right.$ $\{0,1\}))$. For each run, an average of $20 \mathrm{k}$ multihit, multishell measurements were taken.

\section{B. Results versus 2-loop perturbation theory}

In Sec. II, we derived the interface tension at 1-loop, in the continuum and on the lattice. The continuum 2-loop result has been obtained in [8] and in [11], where the running coupling $\tilde{g}(T)$ is chosen such that the 1-loop effects disappear in the renormalization of the coupling in the dimensionally reduced theory. For $\mathrm{SU}(N)$ :

$$
\begin{aligned}
\tilde{\sigma}(T)= & \frac{4 \pi^{2}(N-1)}{3 \sqrt{3 N}} \frac{T^{2}}{\tilde{g}(T)}(1-(15.27853 . . \\
& \left.\left.-\frac{11}{3}\left(\gamma_{E}+\frac{1}{22}\right)\right) \frac{\tilde{g}^{2}(T) N}{(4 \pi)^{2}}\right)
\end{aligned}
$$

with Euler's constant $\gamma_{E}=0.577215 \ldots$ The coupling $\tilde{g}(T)$ is given in [15] as

$$
\left.\tilde{g}^{2}(T) \equiv g_{\overline{M S}}^{2}(\mu)\right|_{\mu=4 \pi T e^{-\left(\gamma_{E}+1 / 22\right)},}
$$

which defines the $\tilde{\Lambda}$ parameter

$$
\tilde{\Lambda}=\frac{e^{\left(\gamma_{E}+1 / 22\right)}}{4 \pi} \Lambda_{\overline{M S}}
$$

With this result it is possible to express the interface tension in (3.6) to subleading order in terms of the lattice bare coupling $g_{\mathrm{LAT}}(T)$. To do that, we use the $\mathrm{SU}(2)$ relation [16]

$$
\Lambda_{\overline{M S}}=19.8228 \Lambda_{\mathrm{LAT}}
$$

to obtain the 1-loop result

$$
\tilde{g}^{2}=g_{\mathrm{LAT}}^{2}\left(1+0.10016 g_{\mathrm{LAT}}^{2}\right),
$$

which is substituted into (3.6) to get

$$
\tilde{\sigma}(T)=\frac{4 \pi^{2}}{3 \sqrt{6}} \frac{T^{2}}{g_{\mathrm{LAT}}(T)}\left(1-0.21467 g_{\mathrm{LAT}}^{2}(T)\right) .
$$

But we want the lattice bare coupling to run with the scale 
$a^{-1}$ instead of $T=\left(a N_{t}\right)^{-1}$ because what is used in a simulation is $\beta=2 N / g_{\mathrm{LAT}}^{2}\left(a^{-1}\right)$. This can be easily translated using the $\beta$ function (3.14). Also the lattice correction factors $C_{l a t}\left(N_{t}\right)$, calculated in Sec. II B and given in Table I, need to be included. Altogether, one gets for SU(2)

$$
\begin{aligned}
\frac{\tilde{\sigma}}{T^{2}}\left(\beta, N_{t}\right)= & C_{\text {lat }}\left(N_{t}\right) \frac{4 \pi^{2}}{3 \sqrt{6}} \frac{\sqrt{\beta}}{2}(1-(0.21467 \\
& \left.\left.+\beta_{0} \log \left(N_{t}\right)\right) \frac{4}{\beta}\right)
\end{aligned}
$$

to subleading order, where $\beta_{0}$ is given in (3.15). Note that we have neglected $\mathcal{O}\left(g^{2}\right)$ corrections in the lattice correction factors $C_{\text {lat }}\left(N_{t}\right)$. How well this (parameter-free!) formula describes the measurements of the interface tension at different $N_{t}$ 's for high $\beta$ can be seen in Fig. 6. The $N_{t}=$ 1 comparison is shown separately because the data do not lie neatly above the other curves but cross right through. We have not performed a similar comparison for $\mathbf{S U}(3)$, but analogous steps predict:

$$
\begin{aligned}
\frac{\tilde{\sigma}}{T^{2}}\left(\beta, N_{t}\right)= & C_{\text {lat }}\left(N_{t}\right) \frac{8 \pi^{2}}{9} \sqrt{\frac{\beta}{6}}(1-(0.34805 \\
& \left.\left.+\beta_{0} \log \left(N_{t}\right)\right) \frac{6}{\beta}\right) .
\end{aligned}
$$

\section{Results versus temperature}

Let us collect our measurements of the interface tension for different values of $\beta$ and $N_{t}$, and correct them with the appropriate lattice correction factor $C_{\text {lat }}\left(N_{t}\right)$, forming $\left(\tilde{\sigma} / T^{2}\right)(T)_{\text {corrected }}=\left(\tilde{\sigma} / T^{2}\right)\left(\beta, N_{t}\right)_{\text {measured }} / C_{\text {lat }}\left(N_{t}\right)$. The bulk of the cutoff effects has been removed with the division by $C_{\text {lat }}\left(N_{t}\right)$, so that the left-hand side can be

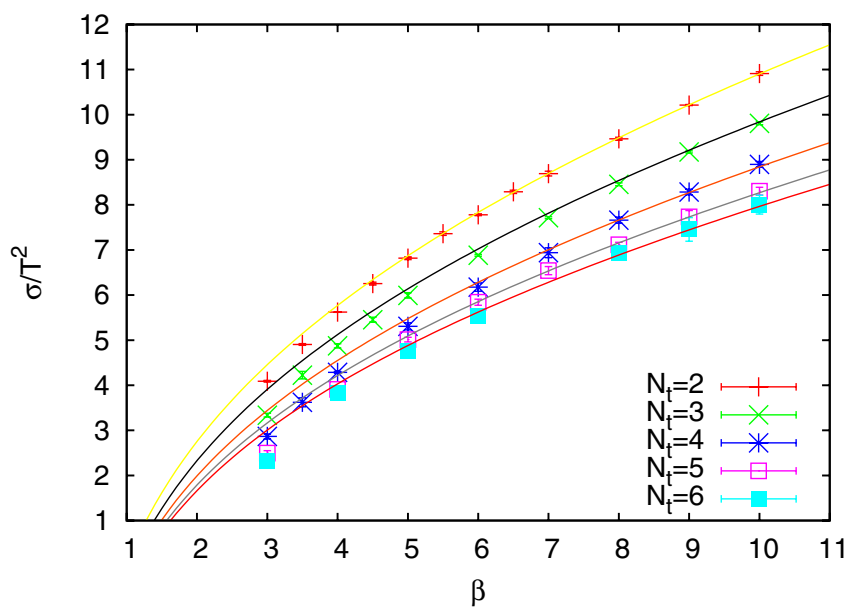

compared with the corresponding continuum quantity evaluated in perturbation theory, as a function of temperature. Since we lack the possibility to set a physical temperature scale (to do that, we would need the nonperturbative $\beta$-function obtained, e.g., from the step scaling function [17]), we calculate the temperature $T=1 /\left(a(\beta) N_{t}\right)$, associated with each value of $\tilde{\sigma} / T^{2}$, using the perturbative 2loop formula for the lattice spacing

$$
a\left(\beta, \Lambda_{\mathrm{LAT}}\right)=\frac{1}{\Lambda_{\mathrm{LAT}}} \exp \left(-\frac{\beta}{8 \beta_{0}}\right)\left(\frac{4 \beta_{0}}{\beta}\right)^{-\beta_{1} /\left(2 \beta_{0}^{2}\right)},
$$

with (here $N=2$ ):

$$
\beta_{0}=\frac{11}{3} \frac{N}{16 \pi^{2}}, \quad \beta_{1}=\frac{34}{3}\left(\frac{N}{16 \pi^{2}}\right)^{2},
$$

which is justified as long as $T \gg \Lambda_{\overline{M S}}$. We want to express the temperature in terms of the parameter $\Lambda_{\overline{M S}}$. One obtains

$$
a\left(\beta, \Lambda_{\overline{M S}}\right)=\frac{19.8228}{\Lambda_{\overline{M S}}} \exp \left(-\frac{\beta}{8 \beta_{0}}\right)\left(\frac{4 \beta_{0}}{\beta}\right)^{-\beta_{1} /\left(2 \beta_{0}^{2}\right)} .
$$

From Eq. (3.6), we know the relation between $\tilde{\sigma} / T^{2}$ and the coupling $\tilde{g}$ at 2-loop order. In [2], Giovannangeli and Korthals Altes show that higher order corrections to Eq. (3.6) are very small. Thus one may hope, that the evaluation of (3.6) for $\mathrm{SU}(2)$ :

$$
\frac{\tilde{\sigma}}{T^{2}}(T)_{\mathrm{GKA}}=\frac{4 \pi^{2}}{3 \sqrt{6}} \frac{1}{\tilde{g}(T)}\left(1-0.16459 \tilde{g}^{2}(T)\right)
$$

is sufficient to describe $\tilde{\sigma} / T^{2}$ over a large range of temperatures. For $\tilde{g}^{2}(T)$ we use the solution to the renormalization group equation to 2-loop:

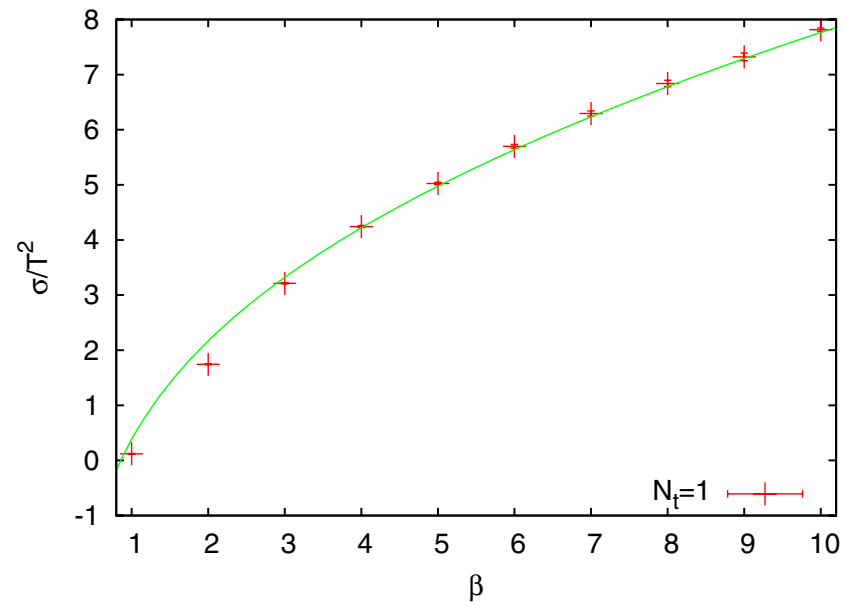

FIG. 6 (color online). The dimensionless ratio of the interface tension over the temperature squared, $\left(\tilde{\sigma} / T^{2}\right)\left(N_{t}, \beta\right)$, versus $\beta$, for different values of $N_{t}$. The data at high $\beta$ are described almost perfectly by the parameter-free formula (3.12), after the lattice correction factors $C_{\text {lat }}\left(N_{t}\right)$ have been inserted. For $N_{t}=1$, the perturbative curve describes the measured data almost perfectly over the whole range $\beta>\beta_{c}$. The reason for this remarkably good agreement is, that the singularity in (3.12) occurs for $\beta=0.8587$, which is fortuitously close to the critical value $\beta_{c}=0.8730$ (2) [19] of $N_{t}=1$. 


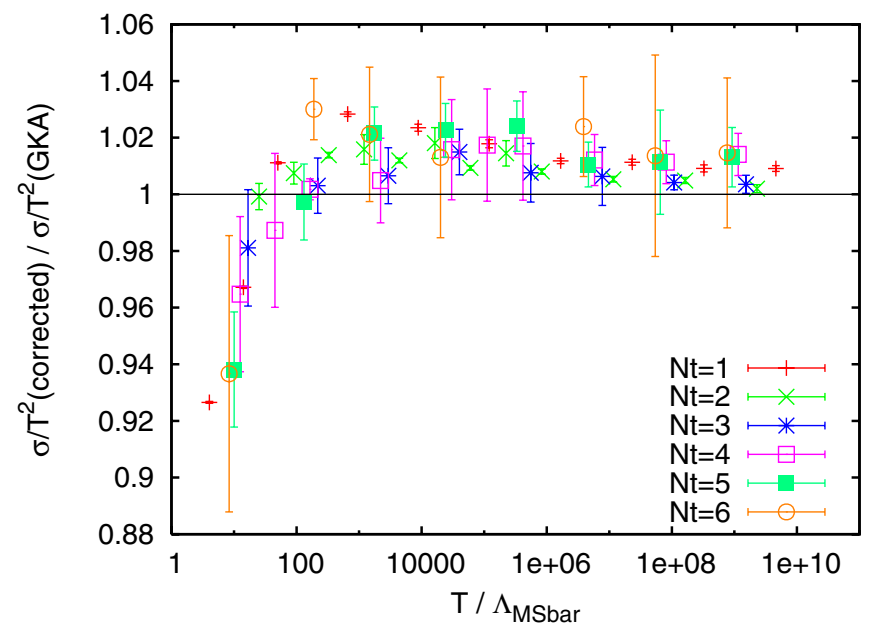

FIG. 7 (color online). The ratio of $\left(\tilde{\sigma} / T^{2}\right)(\hat{T})_{\text {corrected }}$ over $\left(\tilde{\sigma} / T^{2}\right)(\hat{T})_{\mathrm{GKA}} \quad($ Eq. $(3.17))$ versus $\hat{T}=T / \Lambda_{\overline{M S}}$. Deviations from 1 are small $(\sim 2 \%)$, and become appreciable only when $T \lesssim 10 T_{c}$, with $T_{c} / \Lambda_{\overline{M S}}=1.31(8)$ [19].

$$
\tilde{g}^{2}(T)=\frac{1}{\beta_{0} \log \left(T^{2} / \tilde{\Lambda}^{2}\right)+\left(\beta_{1} / \beta_{0}\right) \log \log \left(T^{2} / \tilde{\Lambda}^{2}\right)},
$$

where $\tilde{\Lambda}$ is related to $\Lambda_{\overline{M S}}$ through Eq. (3.8). The perturbative approximation $\left(\tilde{\sigma} / T^{2}\right)(T)_{\mathrm{GKA}}$ (3.17) is compared to our measurements $\left(\tilde{\sigma} / T^{2}\right)(T)_{\text {corrected }}$ in Fig. 7, which shows the ratio $\tilde{\sigma}(T)_{\text {corrected }} / \tilde{\sigma}(T)_{\mathrm{GKA}}$ versus $T / \Lambda_{\overline{M S}}$. As can be seen, the deviations from 1 are remarkably small and remain at the $2 \%$ level down to temperatures $\mathcal{O}(10) T_{c}$. This figure is a beautiful confirmation of the work of Giovannangeli and Korthals Altes.

\section{DISCUSSION}

We have shown that cutoff effects on lattice measurements of the Yang-Mills interface tension (or dual string tension) are large for practical lattice sizes used in Monte Carlo simulations. They do not disappear as $\beta \rightarrow$ $\infty$ as could be naively expected. Table I, which lists these corrections calculated at 1-loop order as a function of the temporal lattice size $N_{t}$ for any $\mathrm{SU}(N)$ group, should be of practical use.

Moreover, we have shown precise agreement between our SU(2) Monte Carlo data and 2-loop continuum perturbation theory, once the correction factors above have been folded in. The ratio of the measured over the perturbative interface tension remains 1 within $\sim 2 \%$, from very high temperatures down to $\mathcal{O}(10) T_{c}$. Our results put the perturbative calculation of the interface tension on firm ground, and show that the perturbative expansion converges well for this quantity, in contrast to the notorious expansion of the pressure [1]. The reason for this remarkable contrast lies presumably in the topological nature of the 't Hooft loop. It naturally encodes the $Z(N)$ center symmetry, which is absent in the perturbative expansion of the pressure.

The small remaining discrepancy between the measured data and perturbation theory can be assigned various causes. We list them in a subjective order of decreasing importance: (i) $\mathcal{O}\left(g^{3}\right)$ correction in $\tilde{\sigma}$ [3]; (ii) $\mathcal{O}\left(g^{2}\right)$ corrections in the lattice correction factors $C_{\text {lat }}$; (iii) 3-loop terms in the lattice $\beta$-function (3.14), which will change the $x$ axis $T / \Lambda_{\overline{M S}}$ in Fig. 7; (iv) 3-loop terms in the running of $\tilde{g}^{2}(T)(3.18) ;(v)$ remaining finite-size effects in the Monte Carlo simulations.

Of course, the low-temperature drop in Fig. 7 is natural. The interface tension is a (dual) order parameter for the phase transition between the cold confining and the hot deconfining phases, which for SU(2) is secondorder. The corresponding correlation length therefore diverges near the critical temperature $T_{c}$ like $\left(T / T_{c}-1\right)^{-\nu}$, with the critical exponent $\nu$ of the $3 \mathrm{~d}$ Ising model as expected from universality [6]. This singularity is not seen by the perturbative calculation. Hence, the ratio $\tilde{\sigma}(T)_{\text {corrected }} / \tilde{\sigma}(T)_{\mathrm{GKA}}$ in Fig. 7 goes to zero like $\left(T / T_{c}-1\right)^{2 \nu}$.

It would be useful to extend our calculation of the lattice correction factors $C_{l a t}$ to order $g^{2}$, for arbitrary $\mathrm{SU}(N)$ group. At this order, one may observe a dependence on the relative $Z(N)$ separation of the two vacua on either side of the interface. This dependence would enter in the comparison of dual $k$-string tensions $\left(\tilde{\sigma}_{k} / \tilde{\sigma}_{1}\right)(T)$ between lattice measurements [10] and continuum perturbation theory [3].

Finally, we became aware of Ref. [18] as this work was being written up. Ref. [18] deals with the same issue of connecting lattice measurements and continuum calculations of dual tensions, for the $3 d$ theory (see Table VIII there). Nevertheless, it seems that some of their analytic results generalize to the $4 d$ case we studied.

\section{ACKNOWLEDGMENTS}

We have benefited from many useful discussions with our colleagues, including Jürg Fröhlich, Pierre Giovannangeli, Chris Korthals Altes, Slavo Kratochvila, Biagio Lucini, Kari Rummukainen, and Michele Vettorazzo.

Note added. - The generalization mentioned above has just been presented in Ref. [20]. Table I there contains similar lattice correction factors as our Table I. The small numerical differences can presumably be assigned to truncation errors in the series used in Ref. [20]. The numerical simulations in the paper focus on ratios of $k$-interface tensions, or rather of their derivatives, in $\mathrm{SU}(N), N>3$. 
[1] K. Kajantie, M. Laine, K. Rummukainen, and Y. Schröder, Phys. Rev. D 67, 105008 (2003).

[2] P. Giovannangeli and C. P. Korthals Altes, Nucl. Phys. B721, 1 (2005).

[3] P. Giovannangeli and C.P. Korthals Altes, Nucl. Phys. B721, 25 (2005).

[4] G. 't Hooft, Nucl. Phys. B138, 1 (1978).

[5] C. Korthals Altes, A. Kovner, and M. A. Stephanov, Phys. Lett. B 469, 205 (1999).

[6] P. de Forcrand, M. D’Elia, and M. Pepe, Phys. Rev. Lett. 86, 1438 (2001).

[7] K. Kajantie, L. Karkkainen, and K. Rummukainen, Nucl. Phys. B333, 100 (1990); Nucl. Phys. B357, 693 (1991); S. Huang, J. Potvin, C. Rebbi, and S. Sanielevici, Phys. Rev. D 42, 2864 (1990); 43, 2056(E) (1991); B. Grossmann, M.L. Laursen, T. Trappenberg, and U.J. Wiese, Nucl. Phys. B396, 584 (1993); R. Brower, S. Huang, J. Potvin, and C. Rebbi, Phys. Rev. D 46, 2703 (1992); Y. Iwasaki, K. Kanaya, L. Karkkainen, K. Rummukainen, and T. Yoshie, Phys. Rev. D 49, 3540 (1994); B. Beinlich, F. Karsch, and A. Peikert, Phys. Lett. B 390, 268 (1997).

[8] T. Bhattacharya, A. Gocksch, C. Korthals Altes, and R. D. Pisarski, Nucl. Phys. B383, 497 (1992).
[9] P. de Forcrand, M. D'Elia, and M. Pepe, Nucl. Phys. B, Proc. Suppl. 94, 494 (2001).

[10] P. de Forcrand, B. Lucini, and M. Vettorazzo, hep-lat/ 0409148.

[11] P. Giovannangeli and C. P. Korthals Altes, Nucl. Phys. B608, 203 (2001).

[12] N. Weiss, Phys. Rev. D 24, 475 (1981).

[13] M. Caselle, M. Hasenbusch, and M. Panero, J. High Energy Phys. 01 (2003) 057.

[14] K. Dietz and T. Filk, Phys. Rev. D 27, 2944 (1983).

[15] S.Z. Huang and M. Lissia, Nucl. Phys. B438, 54 (1995).

[16] A. Hasenfratz and P. Hasenfratz, Phys. Lett. B 93, 165 (1980).

[17] M. Lüscher, R. Sommer, U. Wolff, and P. Weisz, Nucl. Phys. B389, 247 (1993).

[18] C. Korthals Altes, A. Michels, M. A. Stephanov, and M. Teper, Phys. Rev. D 55, 1047 (1997).

[19] J. Fingberg, U.M. Heller, and F. Karsch, Nucl. Phys. B392, 493 (1993).

[20] F. Bursa and M. Teper, J. High Energy Phys. 08 (2005) 060. 